\title{
Computational Sensitivity Analysis on a Mathematical Model of Epileptic Seizures
}

\author{
Karen A. Yokley* ${ }^{*}$, Andrew F. Fischer*, Nicholas S. Luke ${ }^{\dagger}$ and Adrienne Rouiller* \\ *Department of Mathematics and Statistics \\ Elon University, Elon, NC 27244 \\ †Department of Mathematics \\ North Carolina A\&T State University, Greensboro, NC 27411 \\ $\ddagger$ Corresponding Author: kyokley@elon.edu
}

Received: 18 December 2014, accepted: 14 August 2015, published: 26 August 2015

\begin{abstract}
Temporal lobe epilepsy is a serious neurological disorder characterized by complex partial seizures, which are thought to originate in the hippocampus. Ordinary differential equation modeling has been used to describe changes in membrane potential of excitatory and inhibitory cells in order to gain insight into seizure propagation. In the current study, a system of ordinary differential equations based on previous modeling is used with distinct biologically reasonable values for membrane capacitance in order to determine model sensitivity to that parameter. Because delay differential equations are used in the model, sensitivity is investigated computationally by examining the variation in output relative to various inputs. Membrane capacitance was found to affect model predictions and whether groups of cells exhibited the same behavior after a certain period of time. Hence, membrane capacitance is a critical parameter when modeling changes in membrane potential and should be incorporated clearly. Changes in model output as a result of changes in a time delay parameter are also investigated.
\end{abstract}

Keywords-epilepsy, membrane potential, sensitivity analysis, mathematical modeling

\section{INTRODUCTION}

Temporal lobe epilepsy is a serious neurological disorder characterized by complex partial seizures, which are thought to originate in the hippocampus [1], [2], [3], [4], [5]. The mechanism of the propagation of these seizures is not completely understood, and there is no single pathology even within temporal lobe epilepsy [2]. Surgical treatments are often recommended and beneficial for patients with epilepsy [5], but the success of these treatments is dependent on the completeness of the tissue removal and the surgical approach [6], [7], [8], [9] as well as a strong understanding of a patient's clinical history [10]. At this time, surgical techniques may be the most effective treatment strategies from both cost and outcome perspectives [11], [12]. Surgical techniques carry inherent dangers and mathematical modeling may aid in developing alternative treatment strategies with positive outcomes [13], [14].

Several studies have investigated seizures and other behavior related to epileptic events using computer simulations [15], [16], [17], [18], [19], Markov processes [20], [21], differential equations [22], [23], [24], [25], [26], [27], [28], or other 
modeling techniques [14], [29], [30], [31], [32]. Much of the differential equation modeling incorporates changes in membrane potential and is based on a model by Hodgkin and Huxley [33], [34]. Understanding how membrane potentials in excitatory cells change over time may help determine how seizures propagate and hence, how to better prevent such seizures. Because epilepsy is a disease that involves the recurrence of seizures, the underlying dynamics of the disease over time are crucial to consider when developing treatment strategies [13]. Ordinary differential equation models specifically describe dynamics over time and therefore may be especially useful. Several models have focused on the excitatory network thought to be involved in seizure propagation [24] and the behavior of both excitatory and inhibitory neurons [15], [22], [23], [25], [26], [27], [28], [29]. In order to be more representative of the physiological system being modeled, some models incorporate a lattice of multiple nodes or clusters of neurons whose behavior are governed by specific equations [23], [25].

Often in modeling, fixed parameters are based on actual measurements. One parameter used in modeling based on the Hodgkin and Huxley model [34] is membrane capacitance. Most plasma membranes have a capacitance around $1 \mu \mathrm{F} / \mathrm{cm}^{2}$ [35] which suggests that a value of $C=1$ is reasonable. Similar models have explicitly used $C=1$ [23], but there are others that use $C=1$ but do not explicitly include the parameter [24], [25]. However, a value close to (but not equal to) 1 may result in different model output than a value of exactly 1 . The study in Gentet et al. [36] looked specifically at membrane capacitance in different neurons. All of their values were indeed around 1, but those values for hippocampal neurons (from eight Wistar rats) were $0.92 \pm 0.08 \mu \mathrm{F} / \mathrm{cm}^{2}$. The other neuron mean values ranged from 0.85 to 1.06 $\mu \mathrm{F} / \mathrm{cm}^{2}$. Additionally, the measured value of membrane capacitance in neurons may depend greatly on the measurement techniques used [37]. Hence, a local sensitivity analysis on the model in the current study is performed to ascertain the effect of changes in $C$ on the model output. If the model output changes greatly with small changes in the value of $C$, this parameter is critical to include when making model predictions or modifying the equations.

In the current study, a previously developed model from Larter et al. [25] is modified to be more defined with respect to units and to be more consistent with previous literature. Additionally, the revised model incorporates a more specific time delay. In the course of modifying the model from Larter et al. [25], a parameter denoting membrane capacitance is reintroduced in the model. This parameter, referred to as $C$, is expected to have a value close to $1 \mu \mathrm{F} / \mathrm{cm}^{2}$. A sensitivity analysis is performed in order to determine if values of $C$ that are close to but not equal to one will have a large impact on model predictions. Because the model involves delay differential equations, the sensitivity investigations will be performed computationally. The impact of small changes in the value of $C$ are important since the Larter model essentially eliminates the parameter $C$ by setting it equal to one [25]. Hence, this study is intended to model membrane potential changes in the hippocampus using foundational equations [34], [38] in context with the addition of inhibitory neurons in a similar way to the study in Larter et al. [25]. Further, this study investigates the importance on the accuracy of the value of the membrane capacitance parameter when making predictions using this model. The parameter for time delay is also varied in the process of the investigation.

\section{Materials And Methods}

\section{A. Model Structure and Development}

The study in Larter et al. [25] used a system of three ordinary differential equations to describe membrane potentials of excitatory and inhibitory neurons in each node of a $36 \times 36$ lattice. The lattice was intended to represent the CA 3 region of the hippocampus because this region is a common location of the epileptic focus. The model in Larter et al. [25] is based on equations describing voltage 
K. A. Yokley et al., Computational Sensitivity Analysis on a Mathematical Model ...

oscillations in the barnacle giant muscle fiber [38] which itself is based on a model by Hodgkin and Huxley [34]. The foundation for the pyramidal (excitatory) nerve equations in Larter et al. [25] is a model of a single voltage-dependent conductance in which the calcium $\left(\mathrm{Ca}^{2+}\right)$ system is assumed to be much faster than the potassium $\left(\mathrm{K}^{+}\right)$system as described in the study by Morris and Lecar [38]. Larter et al. [25] adds one additional differential equation in each node for the inclusion of inhibitory neurons.

The units of the model in Larter et al. [25] are primarily arbitrary, but the units in the source equations are specifically defined. The models in earlier studies [34], [38] used a particular parameter, $C$ (membrane capacitance, $\mu \mathrm{F} / \mathrm{cm}^{2}$ ), but the model in Larter et al. [25] essentially eliminates this parameter by setting $C=1$, which is a reasonable value [35], [36] and has been used in other similar models [23]. Additionally, Larter et al. [25] did not use delay differential equations but did incorporate a time difference when modeling $\mathrm{K}^{+}$transfer from surrounding nodes.

The model in the current study is similar in concept to the model in Larter et al. [25] in that it incorporates the effect of inhibitory neurons, but the current model uses two equations per node for this effect instead of one. These equations are changed in order to model the membrane potential of inhibitory cells the same way the potential of the excitatory neurons are described in Larter et al. [25] and to be more similar to previous models [23], [34], [38]. Sodium channels, $\mathrm{Na}^{+}$, are represented (instead of calcium channels) as in [34]. Additionally, a continuous delay is used when describing $\mathrm{K}^{+}$transfer from surrounding nodes, and membrane capacitance $(C)$ is reintroduced as an explicit parameter. Both of these changes were made to make the model more physically representative. The updated equations for each
TABLE I

DESCRIPTIONS AND VALUES OF PARAMETERS USED IN EQUATIONS (17)-(4). ALL VALUES BELOW WERE USED IN THE MODEL IN LARTER $e t$ al. [25] EXCEPT FOR $V_{L}$ (NOTED WITH $*$ BELOW), WHICH WAS ALTERED TO MAKE MODEL OUTPUT MORE CYCLIC.

\begin{tabular}{|l|l|l|l|}
\hline & Units & Description & Value \\
\hline \hline$g_{N a}^{V}$ & $m S / \mathrm{cm}^{2}$ & $\begin{array}{l}\text { total conductance for } \mathrm{Na}^{+} \\
\text {channels }\end{array}$ & 1.1 \\
\hline$g_{K}^{V}$ & $m S / \mathrm{cm}^{2}$ & $\begin{array}{l}\text { total conductance for } \mathrm{K}^{+} \\
\text {channels }\end{array}$ & 2.0 \\
\hline$g_{L}^{V}$ & $m S / \mathrm{cm}^{2}$ & $\begin{array}{l}\text { total conductance of leakage } \\
\text { channels }\end{array}$ & 0.5 \\
\hline$g_{N a}^{Z}$ & $m S / \mathrm{cm}^{2}$ & $\begin{array}{l}\text { total conductance for } \mathrm{Na}^{+} \\
\text {channels }\end{array}$ & set as $g_{N a}^{V}$ \\
\hline$g_{K}^{Z}$ & $m S / \mathrm{cm}^{2}$ & $\begin{array}{l}\text { total conductance for } \mathrm{K}^{+} \\
\text {channels }\end{array}$ & set as $g_{K}^{V}$ \\
\hline$g_{L}^{Z}$ & $m S / \mathrm{cm}^{2}$ & $\begin{array}{l}\text { total conductance of leakage } \\
\text { channels }\end{array}$ & set as $g_{L}^{V}$ \\
\hline$V_{N a}$ & $m V$ & $\begin{array}{l}\text { equilibrium potential for } \\
\text { Na }\end{array}$ & 1 \\
\hline$V_{L}$ & $m V$ & $\begin{array}{l}\text { equilibrium potential for } \\
\text { leak conductances }\end{array}$ & $0.01^{*}$ \\
\hline$Z_{N a}$ & $m V$ & $\begin{array}{l}\text { equilibrium potential for } \\
\text { Na }\end{array}$ & set as $V_{N a}$ conductances \\
\hline$Z_{L}$ & $m V$ & $\begin{array}{l}\text { equilibrium potential for } \\
\text { leak conductances }\end{array}$ & set as $V_{L}$ \\
\hline applied current & $\mu A / \mathrm{cm}^{2}$ & 0.30 \\
\hline
\end{tabular}

node are presented below:

$$
\begin{aligned}
\frac{d V_{i}}{d t}= & \frac{1}{C}\left[-g_{N a}^{V} m_{\infty}\left(V_{i}\right)\left(V_{i}-V_{N a}\right)\right. \\
& \left.-g_{K}^{V} W_{i}\left(V_{i}-V_{i}^{K}\right)-g_{L}^{V}\left(V_{i}-V_{L}\right)+I\right] \\
& -\alpha_{i n h}\left(Z_{i}\right) Z_{i} \\
\frac{d W_{i}}{d t}= & \frac{\left(w_{\infty}\left(V_{i}\right)-W_{i}\right)}{\tau_{w}\left(V_{i}\right)} \\
\frac{d Z_{i}}{d t=} & \frac{1}{C}\left[-g_{N a}^{Z} m_{\infty}\left(Z_{i}\right)\left(Z_{i}-Z_{N a}\right)\right. \\
& \left.-g_{K}^{Z} N_{i}\left(Z_{i}-Z_{i}^{K}\right)-g_{L}^{Z}\left(Z_{i}-Z_{L}\right)+I\right] \\
& +\alpha_{e x c}\left(V_{i}\right) V_{i} \\
\frac{d N_{i}}{d t}= & \frac{\left(n_{\infty}\left(Z_{i}\right)-N_{i}\right)}{\tau_{n}\left(Z_{i}\right)}
\end{aligned}
$$

The states at each node $i$ are $V_{i}$, the membrane potential of the pyramidal cells; $Z_{i}$, the membrane potential of the inhibitory cells; and $W_{i}$ and $N_{i}$, relaxation factors. Descriptions of the parameters (other than $C$ ) and their fixed values are presented in Table 【. 
Equations (1) and (2) are the same as in the Larter model [25] with the parameter for membrane capacitance, $C\left(\mu \mathrm{F} / \mathrm{cm}^{2}\right)$ explicitly included and a temperature scaling factor removed. Equation (1) is directly from the model from Morris and Lecar [38] with an additional loss term, $\alpha_{i n h}\left(Z_{i}\right) Z_{i}$, representing the effect of the inhibitory neurons on the membrane potential of the pyramidal cells. The equation for $Z_{i}$ from Larter et al. [25] was replaced in this study with a differential equation (equation (3) above) that is similar in structure to equation (1). In equation (3), the excitatory effect on the inhibitory cells is modeled through an additive term, $\alpha_{\text {exc }}\left(V_{i}\right) V_{i}$, which is similar to the way that effect was modeled by Larter et al. [25]. The rest of equation (3) is directly from the Morris and Lecar model [38]. Both equations (1) and (3) are based on the Morris and Lecar model of single voltage-dependent conductance in which the $\mathrm{Ca}^{2+}$ system is assumed to be much faster than the $\mathrm{K}^{+}$system [38], and a similar assumption is made here for the $\mathrm{Na}^{+}$ channels. Equation (4) is similar to equation (2) and is added in order to model a relaxation factor for the inhibitory neurons in the same way a similar relaxation factor is modeled for the excitatory neurons.

The additional functions used in equations (1)(4) are defined below:

$$
\begin{aligned}
m_{\infty}\left(X_{i}\right) & =0.5\left[1+\tanh \left(\frac{X_{i}-x_{1}}{x_{2}}\right)\right] \\
w_{\infty}\left(X_{i}\right) & =0.5\left[1+\tanh \left(\frac{X_{i}-x_{3}}{x_{4}}\right)\right] \\
n_{\infty}\left(X_{i}\right) & =w_{\infty}\left(X_{i}\right) \\
\alpha_{\text {exc }}\left(V_{i}\right) & =a_{\text {exc }}\left[1+\tanh \left(\frac{V_{i}-x_{5}}{x_{6}}\right)\right] \\
\alpha_{i n h}\left(Z_{i}\right) & =a_{i n h}\left[1+\tanh \left(\frac{Z_{i}-x_{7}}{x_{6}}\right)\right] \\
\tau_{w}\left(X_{i}\right) & =\left[\cosh \left(\frac{X_{i}-x_{3}}{2 x_{4}}\right)\right]^{-1}=\tau_{n}\left(X_{i}\right)
\end{aligned}
$$

where $X_{i}$ is either $V_{i}$ or $Z_{i}$. The functions defined in equations (5)-(10) were used the Larter model
[25] as taken from the model by Morris and Lecar [38]. Other studies have used different functions that are also sigmoidal [23], [24], [27]. The notation of the constants in equations (5)-(10) has been changed from the Larter model [25] in order to avoid confusion. Lowercase letters are now used so that constants will not be confused with state values at particular nodes. The parameter values used in equations (5)-(10) are presented in Table III. The additional functions added in the current study, $\tau_{n}\left(Z_{i}\right)$ and $n_{\infty}\left(Z_{i}\right)$, are defined identically to functions with a similar purpose. Equations (5) and (6) represent the fraction of open voltage-regulated $\mathrm{Na}^{+}$channels, and the effect of excitation or inhibition is modeled in a similar way in equations (8) and (9). Equations (5), (6), and (10) were also used in the Larter model [25] and taken directly from the study by Morris and Lecar [38]. More information on the functions used in equations (5)-(10) can be found in the study by Larter et al. [25].

The model in Larter et al. [25] did not use delay differential equations but did incorporate a time step when modeling the change in membrane potential due to extracellular potassium. The quantity $V_{i}^{K}$ in the current study and in Larter et al. [25] models how the membrane potential of surrounding nodes affects node $i$. In the current study, however, this effect is modeled using a delay term as described below

$$
X_{i}^{K}=\sum_{j} \frac{X_{j}(t-\tau)}{6}-\frac{1}{2}
$$

where $j$ indicates the number of a node adjacent to node $i$ and $X$ represents either $V$ or $Z$. Each node is assumed to contribute equally to an adjacent node and periodic boundary conditions are used. Hence, $X_{j}(t-\tau) / 6$ is the delayed contribution of node $j$ to node $i$ for either excitatory $(V)$ or inhibitory $(Z)$ neurons. The lattice used in the current study is similar in structure to that used in the Larter et al. study [25] in that it is composed of hexagonal "nodes." The current study uses a smaller lattice as shown in Figure 1. Nodes are numbered beginning with the upper left corner and proceeding left to right and on to additional 


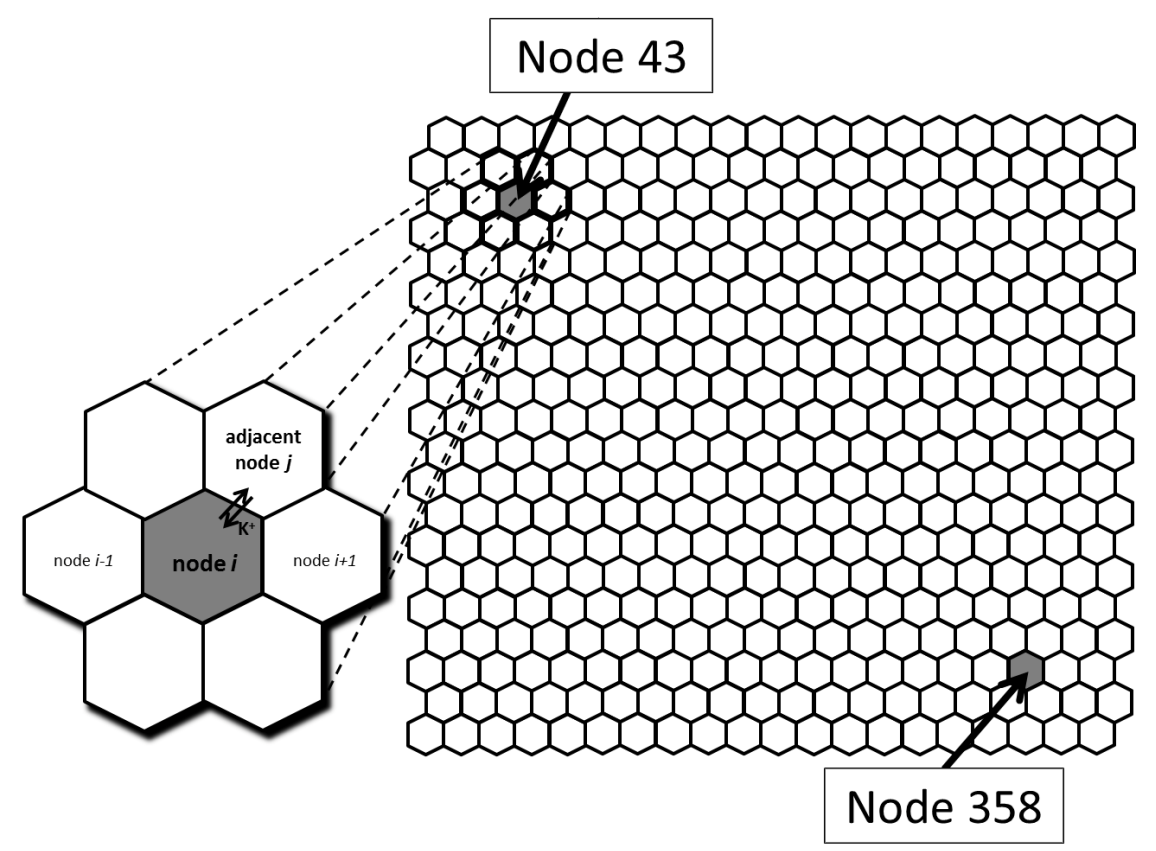

Fig. 1. Illustration of the lattice used in the simulations.

rows. This illustration also includes a closer view of the six nodes surrounding any internal node. Conceptually, the lattice in the current study does differ from that of other studies [23] in that the inhibitory cells are considered to affect other inhibitory nodes surrounding them. The lattice is intended to represent populations of cells (as with [23]) which conforms to the strategy of using variables not at a microscopic but at a macroscopic level as used in the study by Kaneko [39].

Although the authors from Larter et al. [25] did not use $\tau$, their model used an integration time period, $t_{i n t}$, which functioned in a similar capacity. Larter et al. discovered that the lattice became synchronized, i.e., the difference between node values was negligible, if $t_{\text {int }}$ fell below a particular threshold (13.5 in arbitrary units) and used various values of $t_{i n t}$ for their simulations (including a value as high as $t_{\text {int }}=48$ ). Because the current study uses a somewhat different model framework for this time value and because $\tau$ is specifically defined in seconds in the current model, $\tau=1,2,5$, and $10 \mathrm{~s}$ were used for the
TABLE II

DESCRIPTIONS AND VALUES OF PARAMETERS USED IN EQUATIONS (5)-(11). ALL VALUES BELOW WERE USED IN THE MODEL IN LARTER et al [25] ALTHOUGH THE NOTATION HAS CHANGED IN SOME CASES. VARIOUS VALUES WERE USED FOR SOME PARAMETERS IN LARTER et al. [25], BUT ONLY ONE WAS SELECTED FOR THIS INVESTIGATION. THESE VALUES ARE NOTED WITH AN $\operatorname{ASTERIX}(*)$.

\begin{tabular}{|l|l|l|l|}
\hline Param. & Units & Description & Value \\
\hline \hline$x_{1}$ & $m V$ & threshold value for $m_{\infty}$ for $V_{i}$ & -0.01 \\
\hline$x_{2}$ & $m V$ & steepness parameter for $m_{\infty}$ for $V_{i}$ & 0.15 \\
\hline$x_{3}$ & $m V$ & threshold value for $w_{\infty}$ for $V_{i}$ & 0.0 \\
\hline$x_{4}$ & $m V$ & steepness parameter for $w_{\infty}$ for $V_{i}$ & 0.30 \\
\hline$x_{5}$ & $m V$ & threshold value for $\alpha_{e x c}$ & 0.0 \\
\hline$x_{6}$ & $m V$ & $\begin{array}{l}\text { steepness parameter for } \alpha_{e x c} \\
\text { and } \alpha_{i n h}\end{array}$ & $0.6^{*}$ \\
\hline$x_{7}$ & $m V$ & threshold value for $\alpha_{i n h}$ & 0.0 \\
\hline$a_{e x c}$ & & strength of excitatory synapse & $1.0^{*}$ \\
\hline$a_{i n h}$ & & strength of inhibitory synapse & $1.0^{*}$ \\
\hline
\end{tabular}

simulations in order to visually evaluate sensitivity. The leak conductance parameter $V_{L}$ was altered in order to make the solutions exhibit more cyclic behavior, i.e., so that the node potentials did not continue to increase or to decrease. 


\section{B. Computational Methods}

Due to computational restrictions, a smaller lattice size $(20 \times 20)$ was used than in the model from Larter et al. [25]. The differential equations representing the system, (1)-(4) were solved numerically for $C=0.8, C=1.0$, and $C=1.2$ and various values of $\tau$. All simulations were performed using Wolfram Mathematica, version 9.0. The state values for $t<0$ were set equal to their respective initial conditions, and initial conditions did not vary across the lattice. The simulations were run for 350 seconds in order to allow the curves to approach a repeated cycle. The last 150 seconds were used for plotting and are intended to represent a general time period of that length.

The Larter model on which the current study is based investigated the difference in membrane potential between nodes as a measure of synchronization of the lattice [25] because synchronization may be an indication of epileptic phenomenon [1], [2], [32], [27]. A similar analysis was conducted in the current study, and nodes 43 and 358 were chosen for investigation because they were not on the boundary and relatively distant from each other as is shown in Figure 1.

\section{RESUltS}

In order to determine how the reduction in lattice size affected results, computational solutions were generated using a $6 \times 6,10 \times 10$, and $20 \times 20$ lattice and compared. Values of $C=1$, and $\tau=1$ were fixed for these solutions. Nodes 9 and 28, 23 and 78 , and 43 and 358 were investigated for the $6 \times 6,10 \times 10$, and $20 \times 20$ lattices, respectively. Figure 2 contains graphs of the differences in the membrane potentials between the investigated nodes, and differences can be seen in the node differences for both excitatory and inhibitory potentials.

Selected plots of the computational solutions to equations (1)-(4) are presented in Figures 3 . 9. The solution curves correspond to membrane potentials and their sensitivities for nodes 43 and 358 on a $20 \times 20$ lattice. The membrane potentials of excitatory cells at nodes 43 and 358 versus time are presented in Figure 3. (The choice of nodes 43 and 358 for investigation is discussed in Section II-B,) Similarly, the membrane potentials of inhibitory nodes versus time are presented in Figure 4. Three curves are presented in each of these figures which represent solutions using three different values of $C$. Different solution curves are apparent for the three values of $C$, and the largest range of excitatory output is present when $C=0.8$ for most of the investigated values of $\tau$. Additionally the largest magnitude of inhibitory output in the investigation occurs when $C=0.8$ for most investigated values of $\tau$. For cases (such as $\tau=5$ ) when the output ranges are larger for simulations using other values of $C$, there are visible differences in model output for various values of $C$.

An examination of the results for the membrane potential in excitatory neurons (presented in Figure 3) would suggest that changes in the membrane capacitance $(C)$ result in changes in the amplitude and phase of the oscillations. Also, it appears that changes in the delay parameter $(\tau)$ lead to a change in the period/frequency of the oscillations in the membrane potential, as well as the amplitude. Variation in the membrance capacitance and the delay parameter lead to similar changes in the membrane potential in the inhibitory neurons (presented in Figure 4), although changes in the capacitance also seem to affect the baseline of the oscillations.

Differences between predictions at node 43 and node 358 are presented in Figure 5 . These differences are plotted in order to ascertain if the two nodes are predicted to exhibit the same behavior with time. The magnitude of these differences is similar in scale to model output for excitatory and inhibitory cells, which indicates that the model is predicting different values at the investigated nodes when time is fixed.

The membrane potentials for the inhibitory cells shown in Figure 4 and for the pyramidal cells shown in Figure 3 are plotted for individual nodes in Figures 6,96. Each individual graph in 


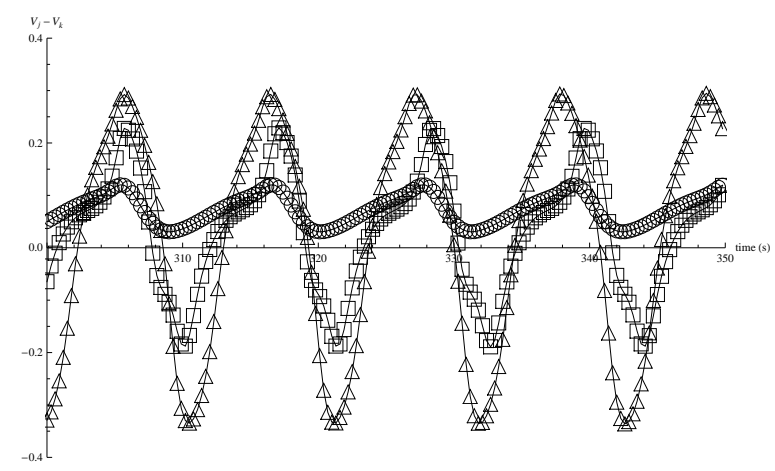

(a) $V_{i}-V_{j}$

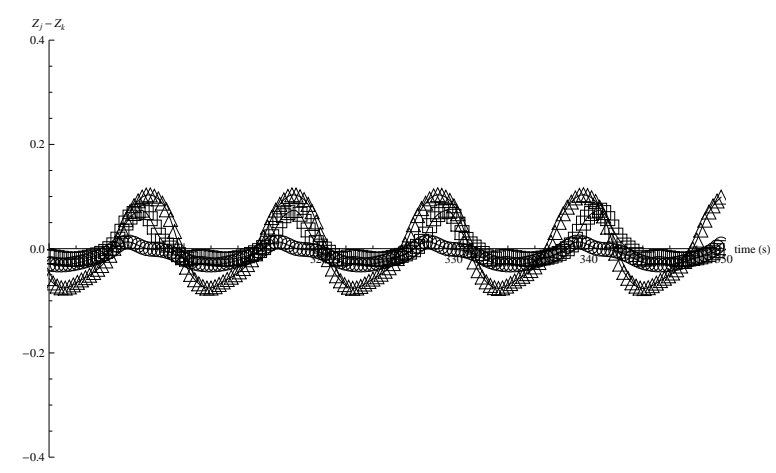

(b) $Z_{i}-Z_{j}$

Fig. 2. Computational solution curves for the differences in membrane potential over different lattice sizes plotted versus time in seconds with $C=1$ and $\tau=1$. (a) Solution curves for $V_{9}-V_{28}$ (circles), $V_{23}-V_{78}$ (squares), and $V_{43}-V_{358}$ (triangles). (b) Solution curves for $Z_{9}-Z_{28}$ (circles), $Z_{23}-Z_{78}$ (squares), and $Z_{43}-Z_{358}$ (triangles).

these figures represents the behavior of inhibitory cells versus the behavior of excitatory cells at an individual node and specific value of membrane capacitance at a particular value of $\tau$. The shapes of these curves remained fairly consistent with changes in the value of $C$ but the cycles were more erratic for increasing values of $\tau$.

Differences in predictions for inhibitory nodes and excitatory nodes are plotted in Figure 10 , and the highest differences (in magnitude) between nodes are presented in Table III for each investigated value of $C$ and $\tau$.
TABLE III

THE MAXIMUM MAGNITUDE DIFFERENCE BETWEEN NODES FOR EACH INVESTIGATION.

\begin{tabular}{|l|c|c|c|}
\hline Type, $\tau$ & $C=0.8$ & $C=1$ & $C=1.2$ \\
\hline \hline$V, \tau=1$ & 0.295 & -0.362 & -0.336 \\
\hline$Z, \tau=1$ & 0.078 & 0.093 & 0.104 \\
\hline$V, \tau=2$ & 0.251 & -0.237 & -0.203 \\
\hline$Z, \tau=2$ & 0.058 & 0.064 & 0.062 \\
\hline$V, \tau=5$ & -0.675 & 0.572 & -0.939 \\
\hline$Z, \tau=5$ & 0.317 & -0.832 & 1.050 \\
\hline$V, \tau=10$ & 0.664 & -0.427 & -0.409 \\
\hline$Z, \tau=10$ & 0.370 & 0.176 & 0.190 \\
\hline
\end{tabular}




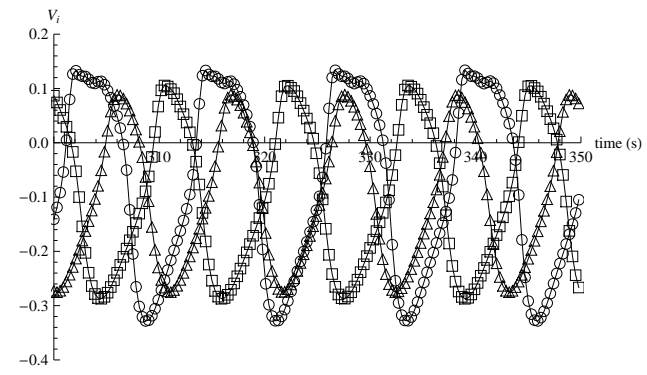

(a) $V_{43}, \tau=1$

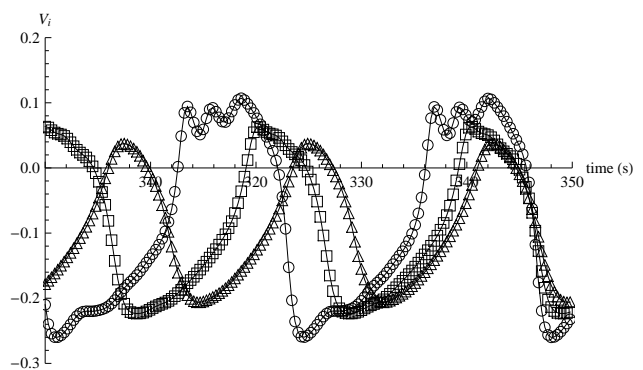

(c) $V_{43}, \tau=2$

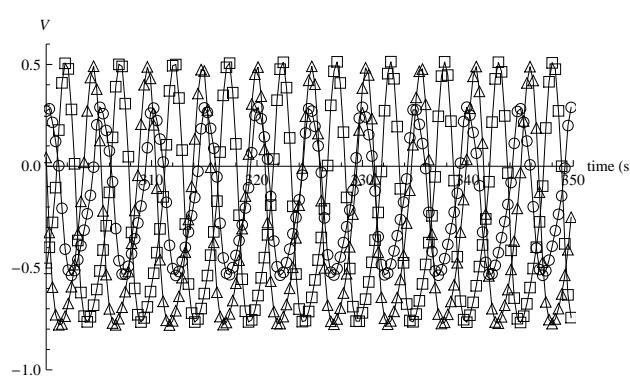

(e) $V_{43}, \tau=5$

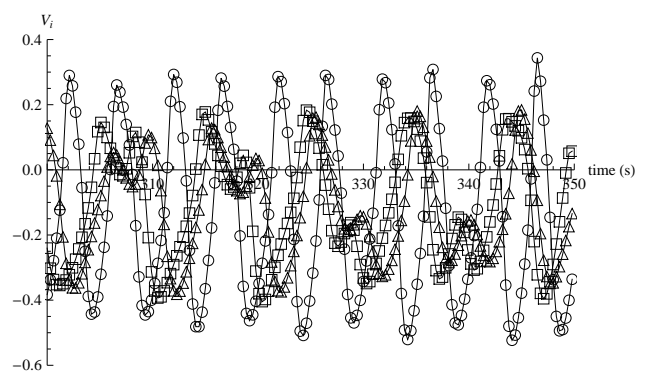

(g) $V_{43}, \tau=10$

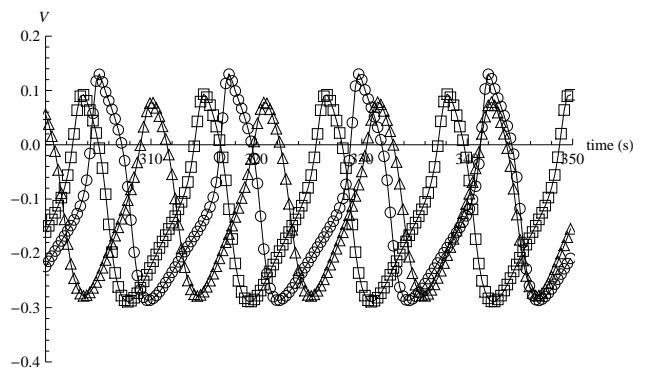

(b) $V_{358}, \tau=1$

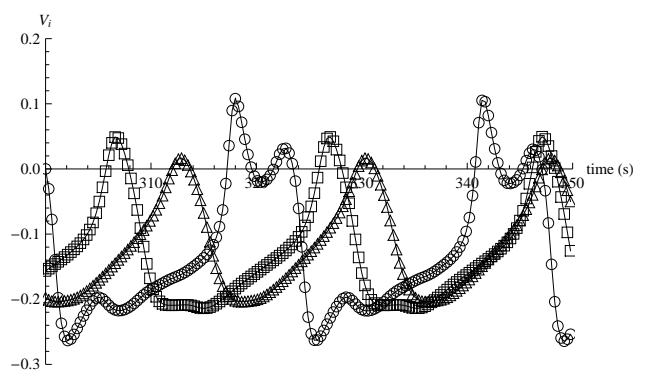

(d) $V_{358}, \tau=2$

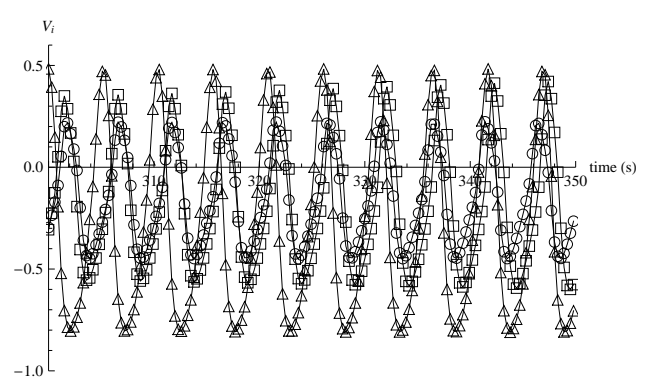

(f) $V_{358}, \tau=5$

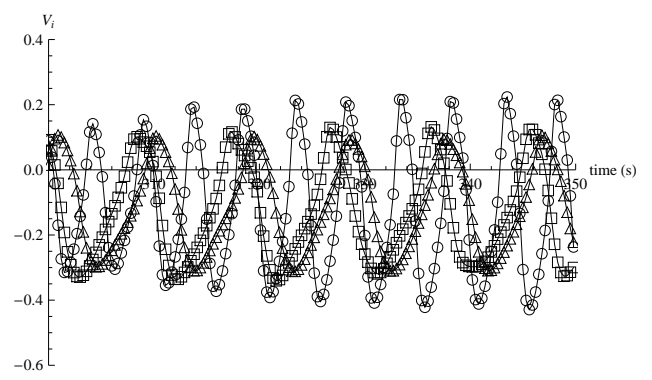

(h) $V_{358}, \tau=10$

Fig. 3. Computational solution curves for $V_{i}$ with $C=0.8$ (circles), $C=1$ (squares), and $C=1.2$ (triangles) versus time in seconds. Solution curves are presented for $V_{43}$ and $V_{358}$ for $\tau=1 \mathrm{~s} \mathrm{(a,b),} \tau=2 \mathrm{~s} \mathrm{(c,d),} \tau=5 \mathrm{~s}(\mathrm{e}, \mathrm{f})$, and $\tau=10 \mathrm{~s}(\mathrm{~g}, \mathrm{~h})$. 


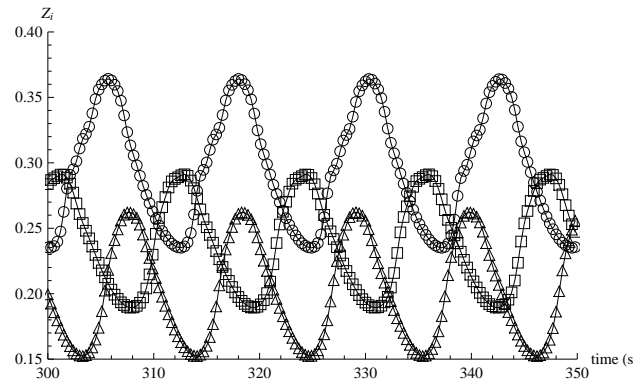

(a) $Z_{43}, \tau=1$

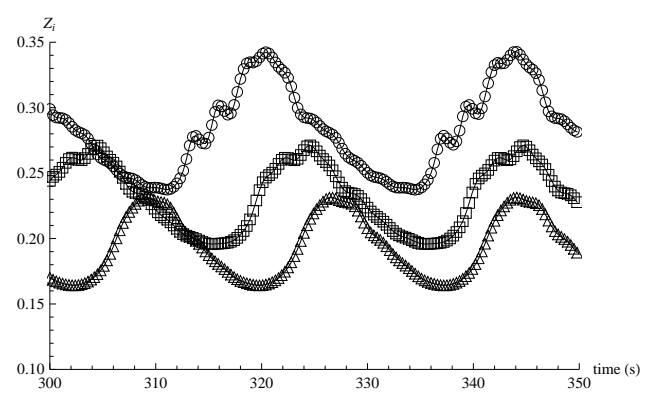

(c) $Z_{43}, \tau=2$

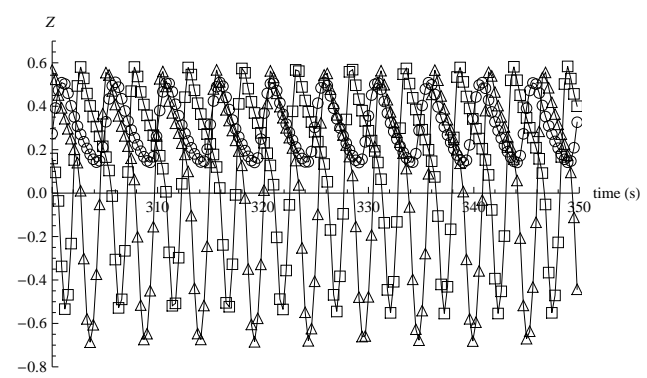

(e) $Z_{43}, \tau=5$

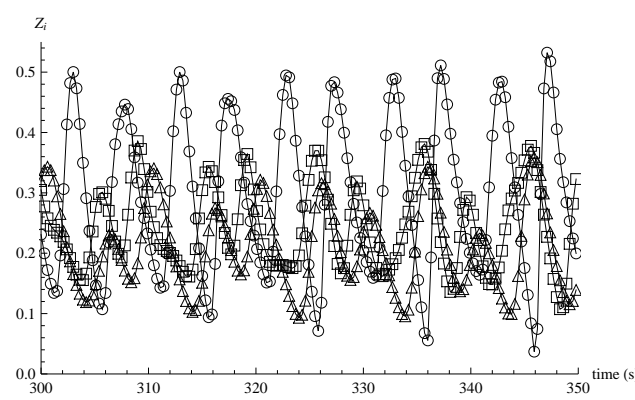

(g) $Z_{43}, \tau=10$

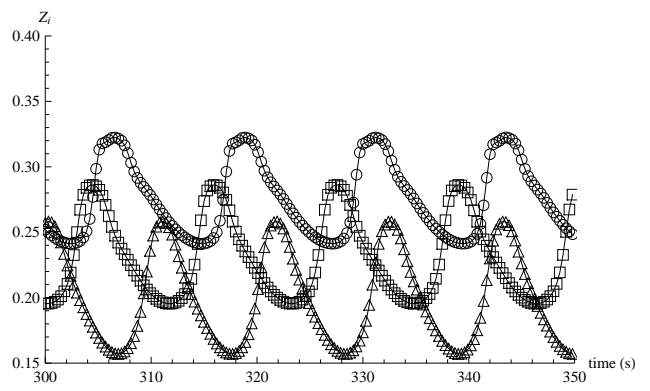

(b) $Z_{358}, \tau=1$

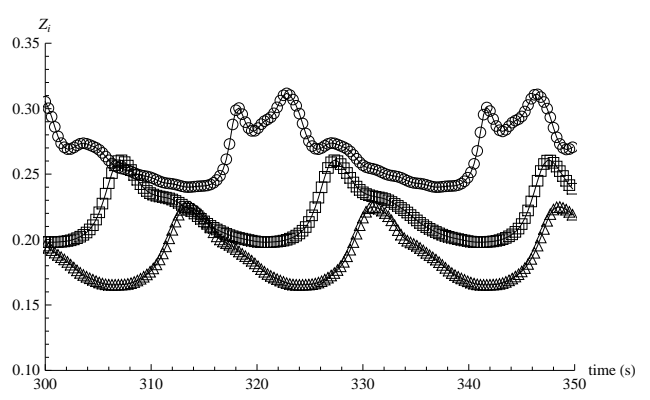

(d) $Z_{358}, \tau=2$

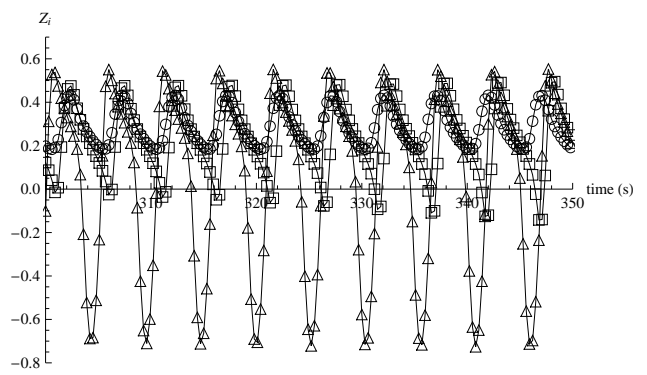

(f) $Z_{358}, \tau=5$

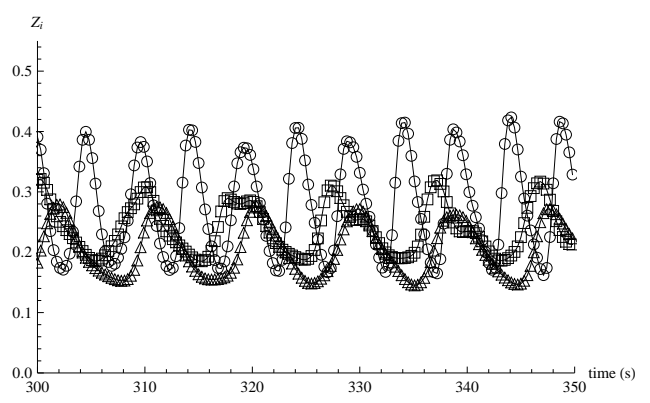

(h) $Z_{358}, \tau=10$

Fig. 4. Computational solution curves for $Z_{i}$ with $C=0.8$ (circles), $C=1$ (squares), and $C=1.2$ (triangles) versus time in seconds. Solution curves are presented for $V_{43}$ and $V_{358}$ for $\tau=1 \mathrm{~s}(\mathrm{a}, \mathrm{b}), \tau=2 \mathrm{~s}(\mathrm{c}, \mathrm{d}), \tau=5 \mathrm{~s}(\mathrm{e}, \mathrm{f})$, and $\tau=10 \mathrm{~s}(\mathrm{~g}, \mathrm{~h})$. 


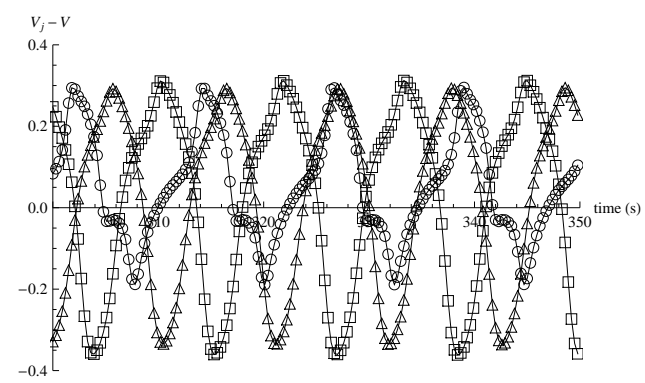

(a) $V_{43}-V_{358}, \tau=1$

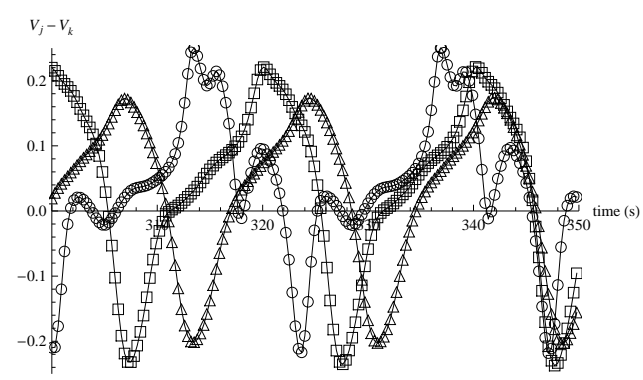

(c) $V_{43}-V_{358}, \tau=2$

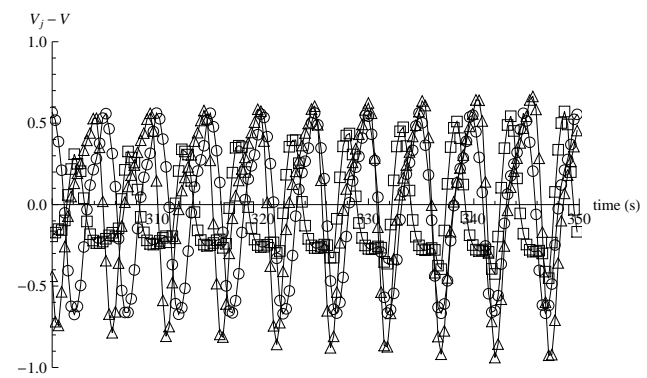

(e) $V_{43}-V_{358}, \tau=5$

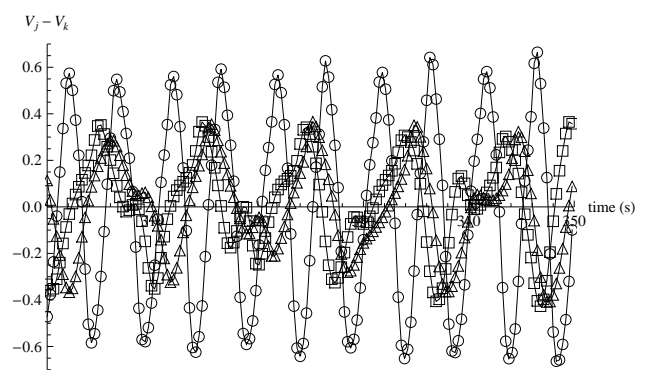

(g) $V_{43}-V_{358}, \tau=10$

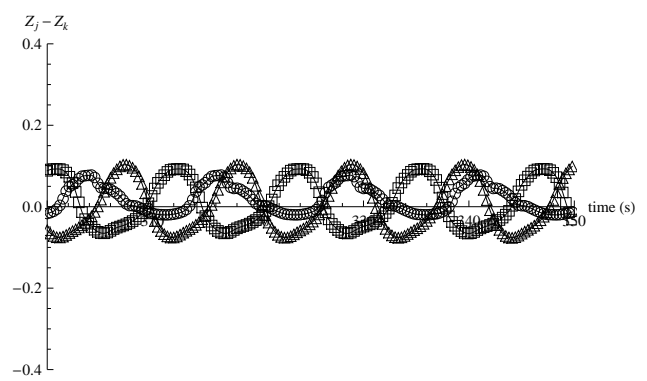

(b) $Z_{43}-Z_{358}, \tau=1$

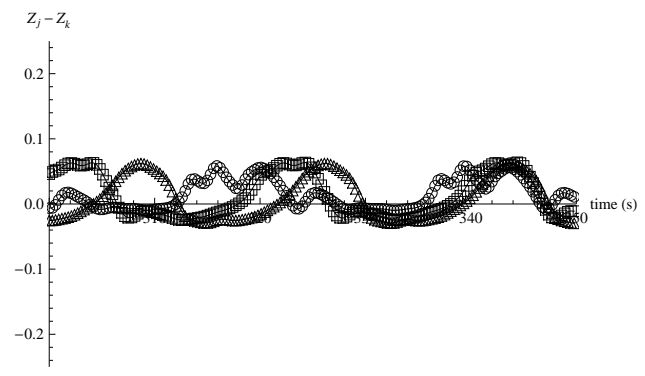

(d) $Z_{43}-Z_{358}, \tau=2$

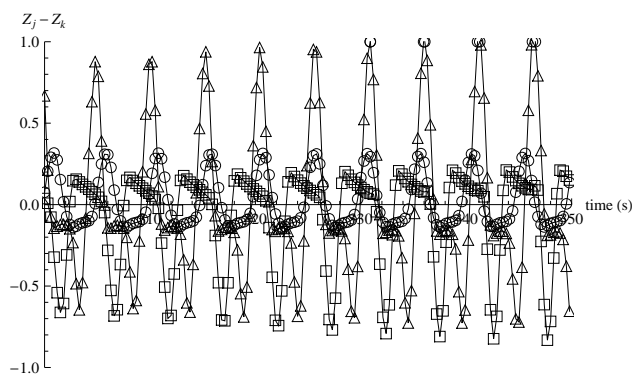

(f) $Z_{43}-Z_{358}, \tau=5$

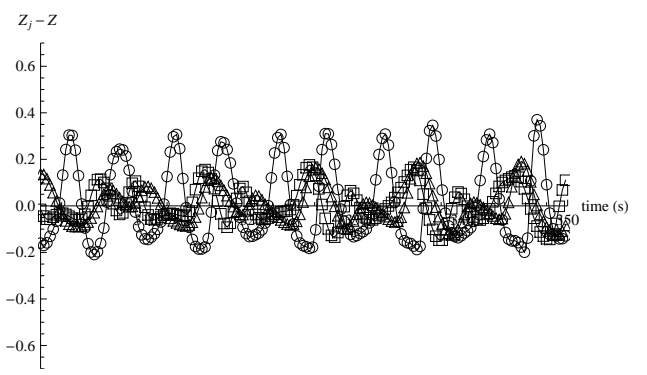

(h) $Z_{43}-Z_{358}, \tau=10$

Fig. 5. Computational solution curves for the differences between nodes with $C=0.8$ (circles), $C=1$ (squares), and $C=1.2$ (triangles) versus time in seconds. Solution curves are presented for $V_{43}-V_{358}$ and $Z_{43}-Z_{358}$ for $\tau=1 \mathrm{~s}(\mathrm{a}, \mathrm{b})$, $\tau=2 \mathrm{~s}(\mathrm{c}, \mathrm{d}), \tau=5 \mathrm{~s}(\mathrm{e}, \mathrm{f})$, and $\tau=10 \mathrm{~s}(\mathrm{~g}, \mathrm{~h})$. 


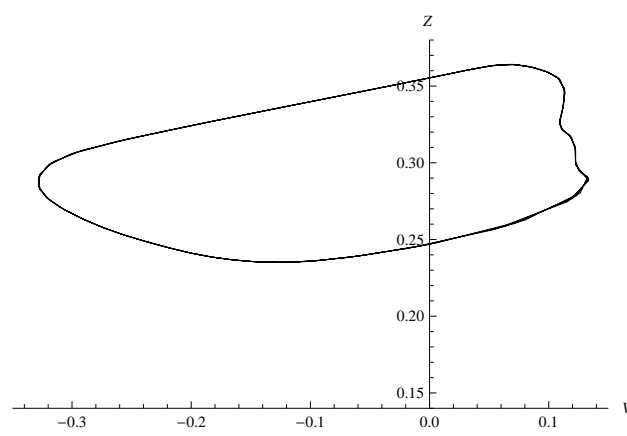

(a) $C=0.8, i=43$

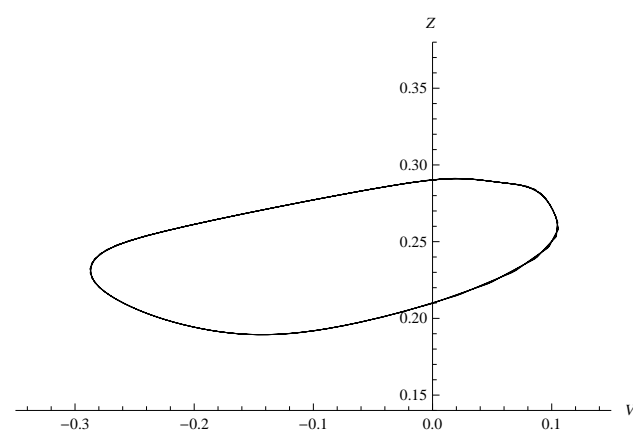

(c) $C=1, i=43$

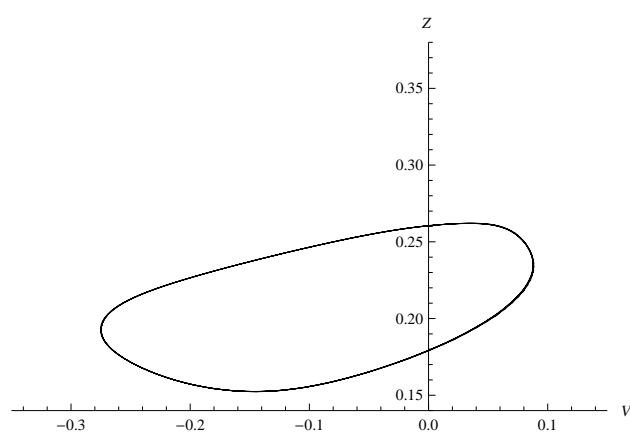

(e) $C=1.2, i=43$

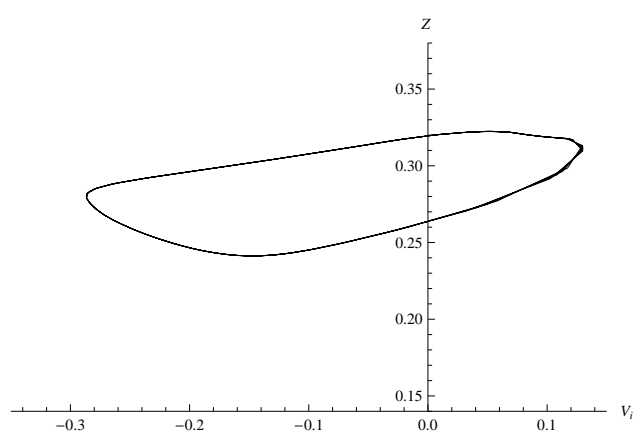

(b) $C=0.8, i=358$

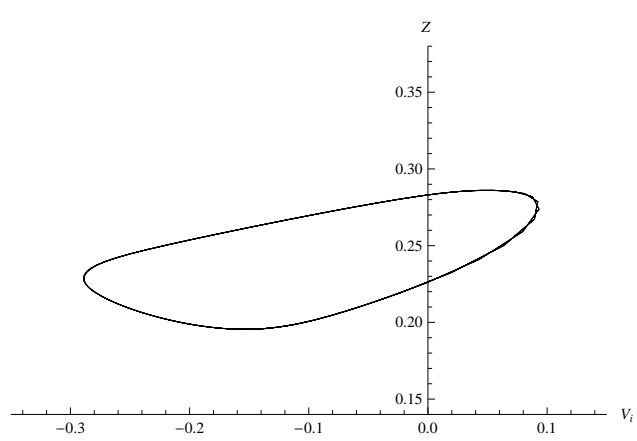

(d) $C=1, i=358$

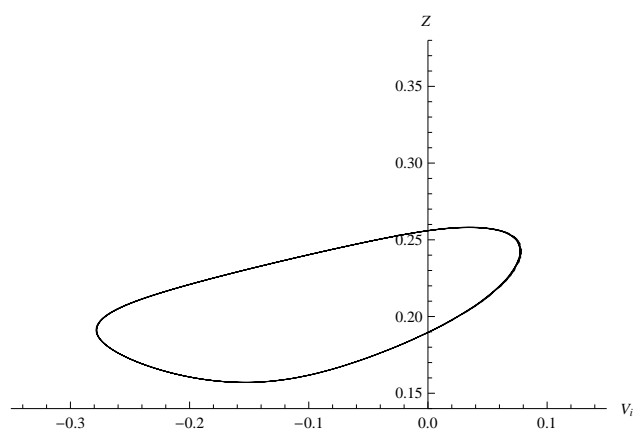

(f) $C=1.2, i=358$

Fig. 6. Computational solution curves for the inhibitory node potentials versus pyramidal node potentials for $\tau=1$ for (a) $C=0.8$ at node $i=43$, (b) $C=0.8$ at node $i=358$, (c) $C=1.0$ at node $i=43$, (d) $C=1.0$ at node $i=358$, (e) $C=1.2$ at node $i=43$, and (f) $C=1.2$ at node $i=358$. The time periods correspond to those used in Figures 3 , 4, and 5 


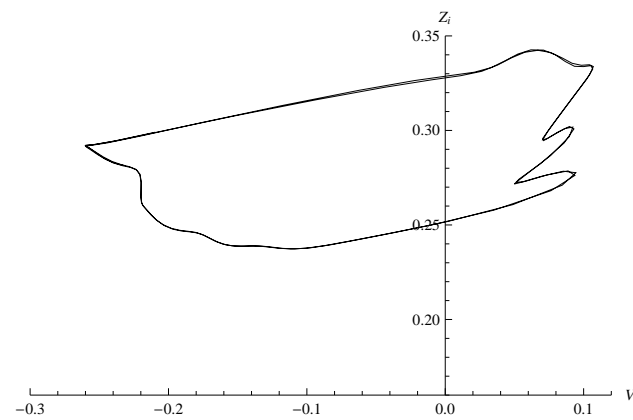

(a) $C=0.8, i=43$

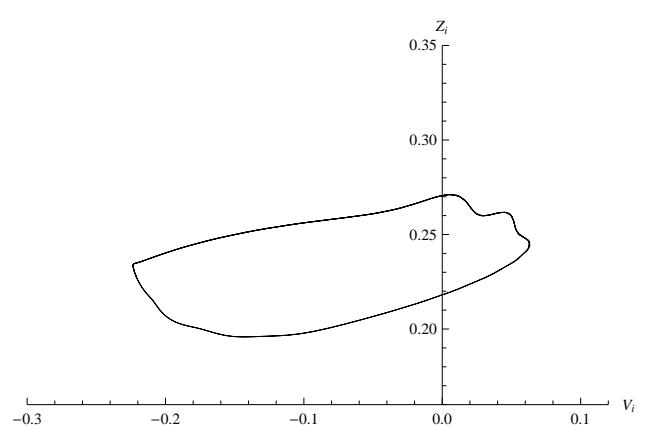

(c) $C=1, i=43$

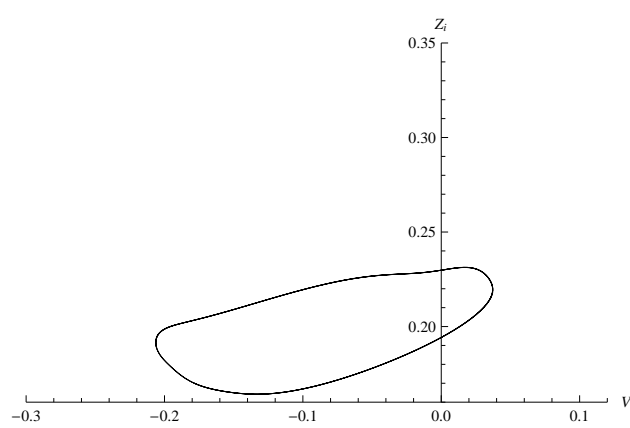

(e) $C=1.2, i=43$

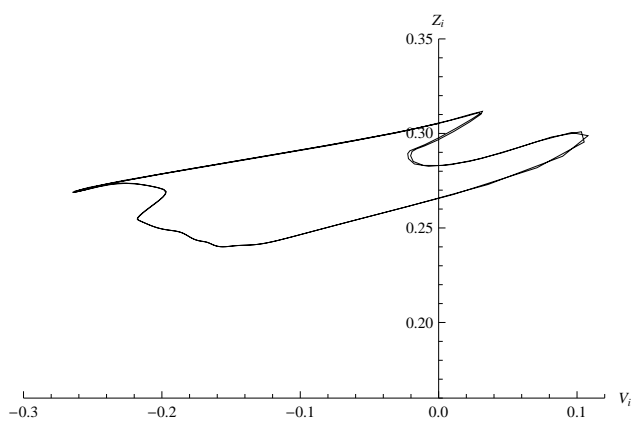

(b) $C=0.8, i=358$

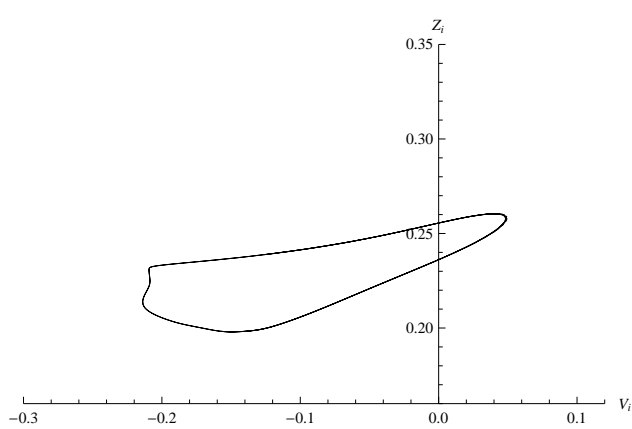

(d) $C=1, i=358$

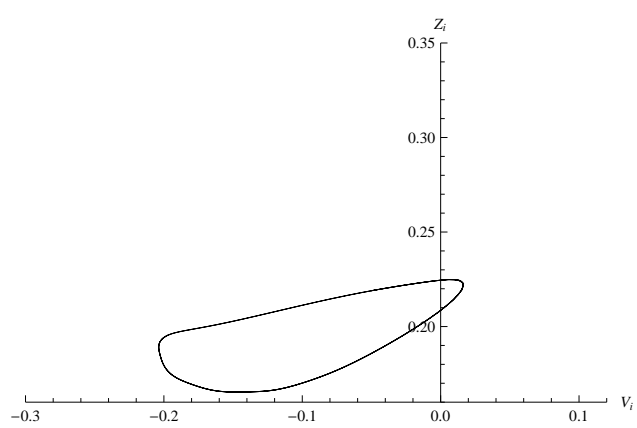

(f) $C=1.2, i=358$

Fig. 7. Computational solution curves for the inhibitory node potentials versus pyramidal node potentials for $\tau=2$ for (a) $C=0.8$ at node $i=43$, (b) $C=0.8$ at node $i=358$, (c) $C=1.0$ at node $i=43$, (d) $C=1.0$ at node $i=358$, (e) $C=1.2$ at node $i=43$, and (f) $C=1.2$ at node $i=358$. The time periods correspond to those used in Figures 3, 4, and 5 


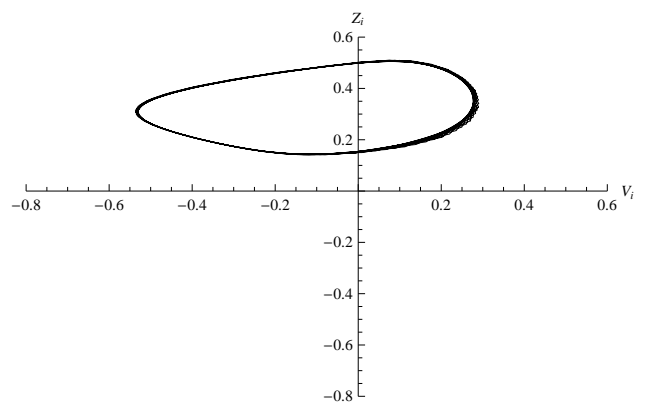

(a) $C=0.8, i=43$

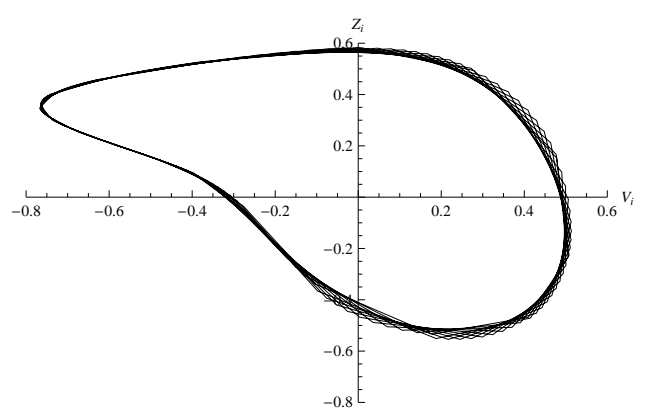

(c) $C=1, i=43$

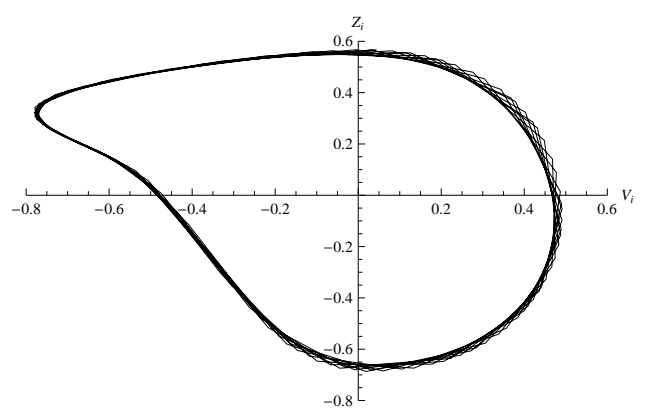

(e) $C=1.2, i=43$

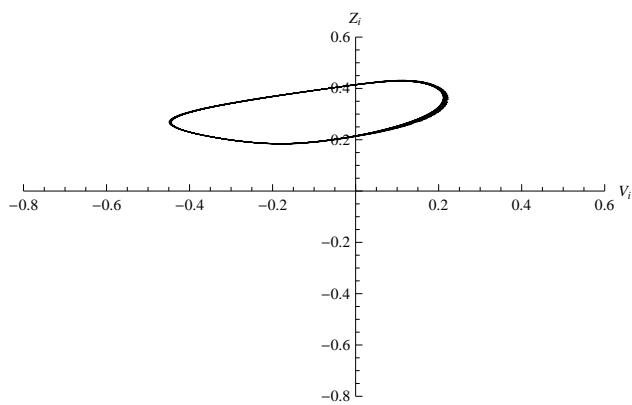

(b) $C=0.8, i=358$

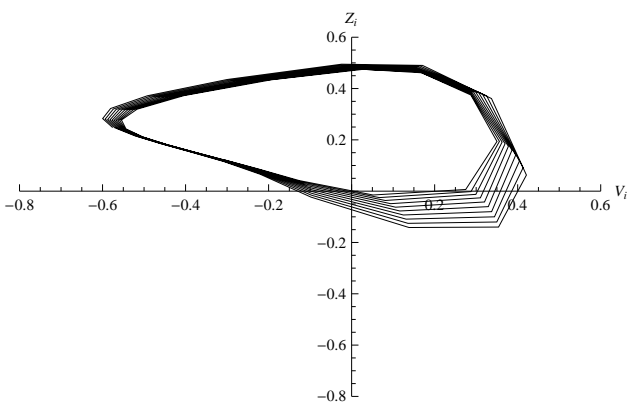

(d) $C=1, i=358$

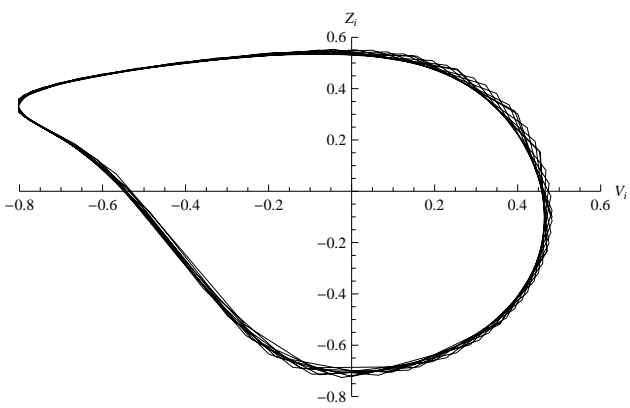

(f) $C=1.2, i=358$

Fig. 8. Computational solution curves for the inhibitory node potentials versus pyramidal node potentials for $\tau=5$ for (a) $C=0.8$ at node $i=43$, (b) $C=0.8$ at node $i=358$, (c) $C=1.0$ at node $i=43$, (d) $C=1.0$ at node $i=358$, (e) $C=1.2$ at node $i=43$, and (f) $C=1.2$ at node $i=358$. The time periods correspond to those used in Figures 3 , 4 and 5 


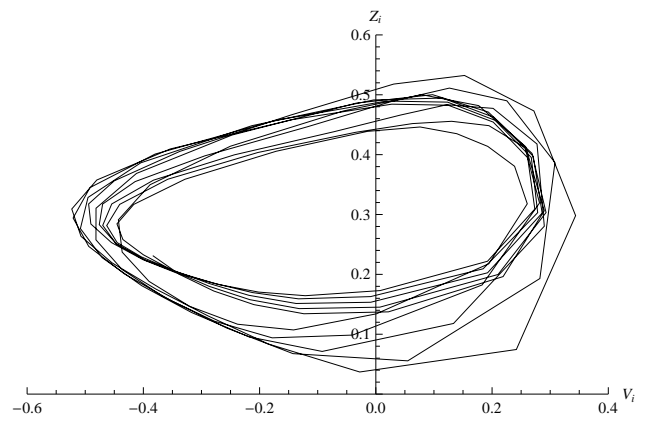

(a) $C=0.8, i=43$

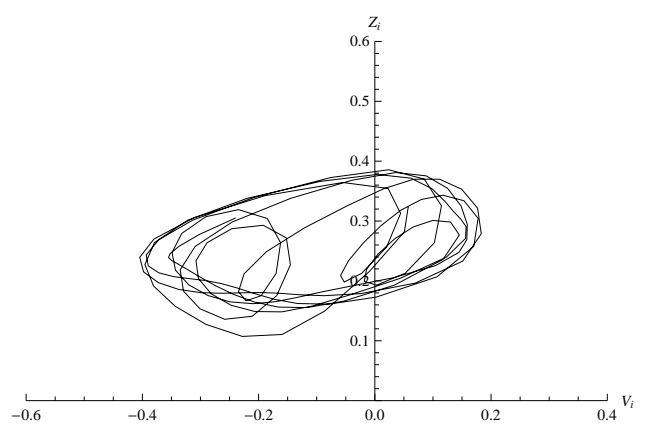

(c) $C=1, i=43$

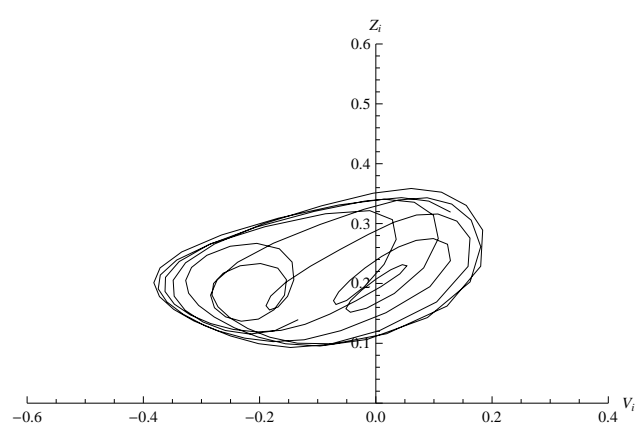

(e) $C=1.2, i=43$

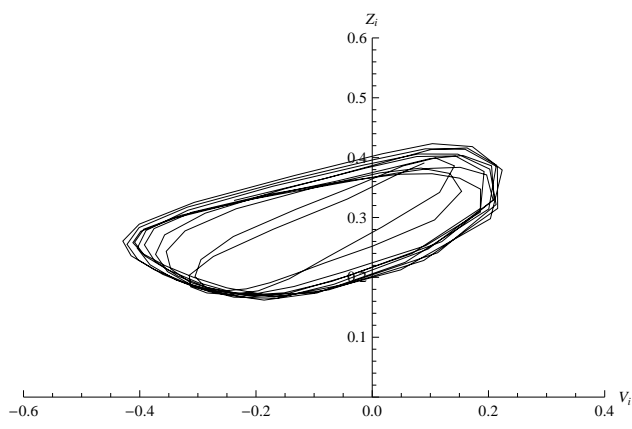

(b) $C=0.8, i=358$

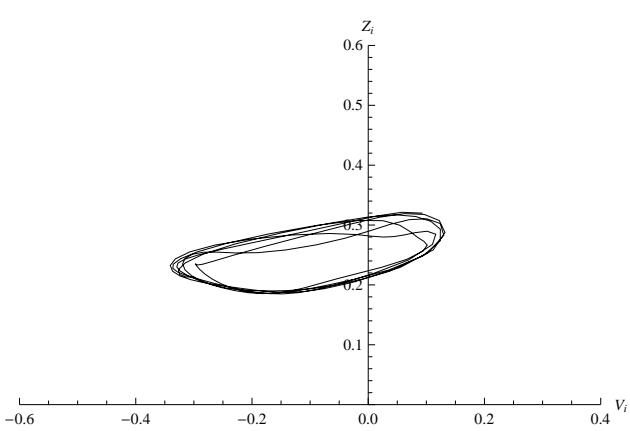

(d) $C=1, i=358$

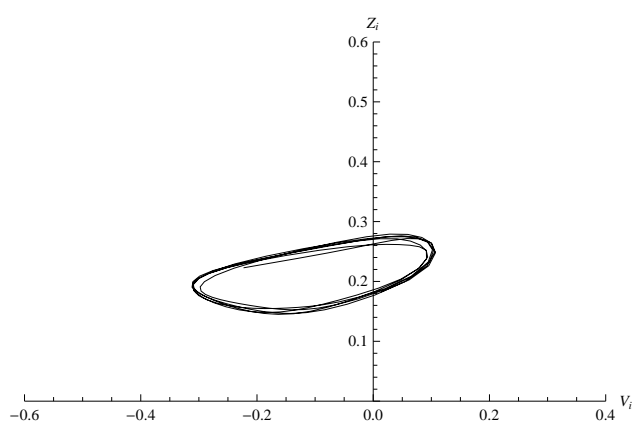

(f) $C=1.2, i=358$

Fig. 9. Computational solution curves for the inhibitory node potentials versus pyramidal node potentials for $\tau=10$ for (a) $C=0.8$ at node $i=43$, (b) $C=0.8$ at node $i=358$, (c) $C=1.0$ at node $i=43$, (d) $C=1.0$ at node $i=358$, (e) $C=1.2$ at node $i=43$, and (f) $C=1.2$ at node $i=358$. The time periods correspond to those used in Figures 3 , 4 , and 5 


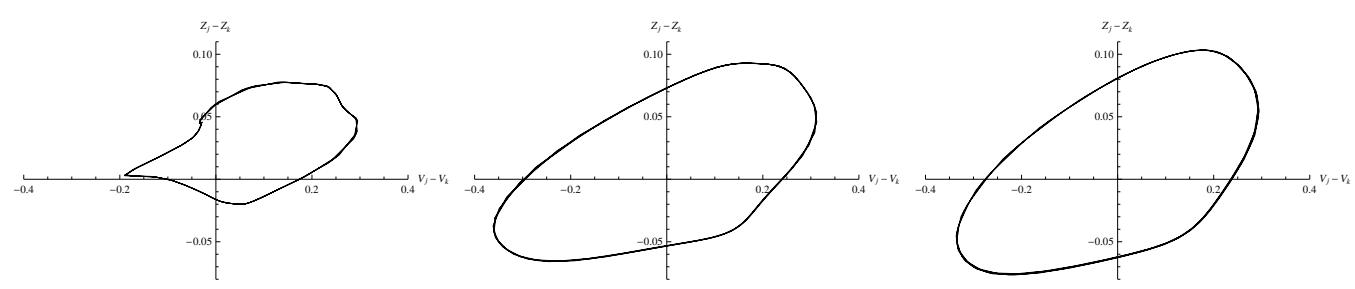

(a) $C=0.8, \tau=1$

(b) $C=1, \tau=1$

(c) $C=1.2, \tau=1$

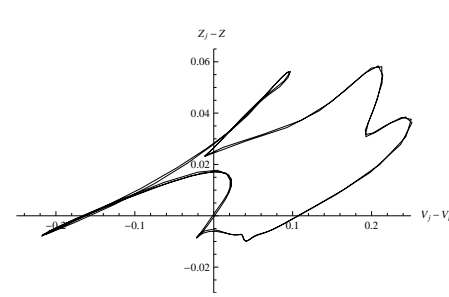

(d) $C=0.8, \tau=2$

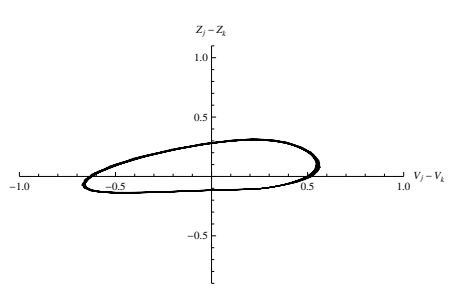

(g) $C=0.8, \tau=5$

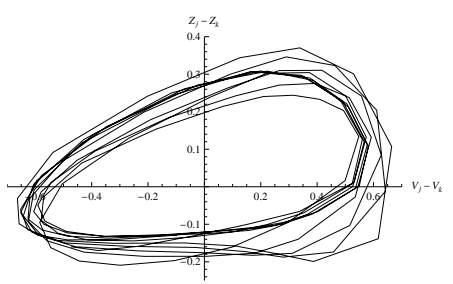

(j) $C=0.8, \tau=10$

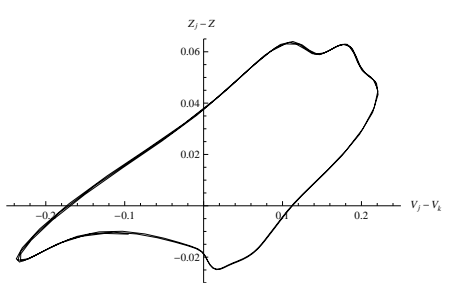

(e) $C=1, \tau=2$

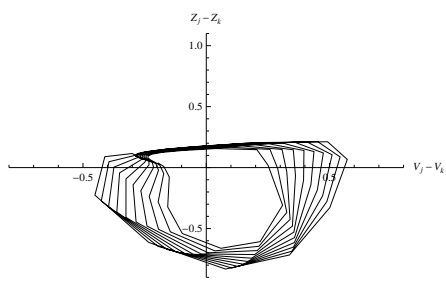

(h) $C=1, \tau=5$

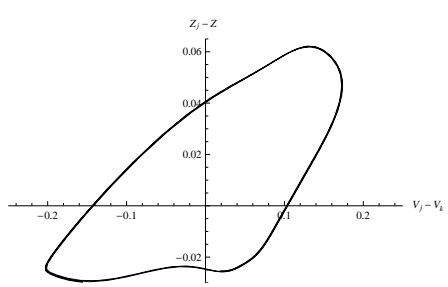

(f) $C=1.2, \tau=2$

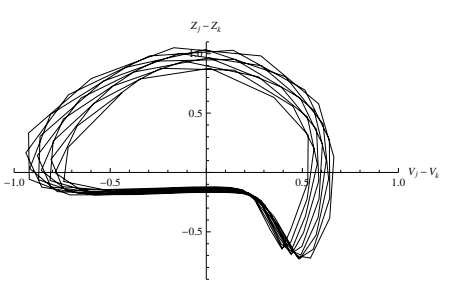

(i) $C=1.2, \tau=5$

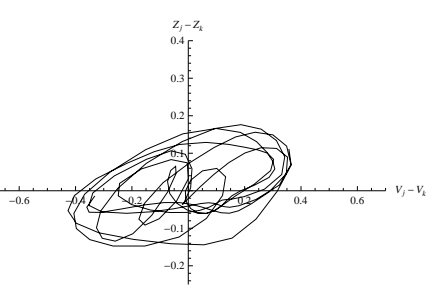

(k) $C=1, \tau=10$

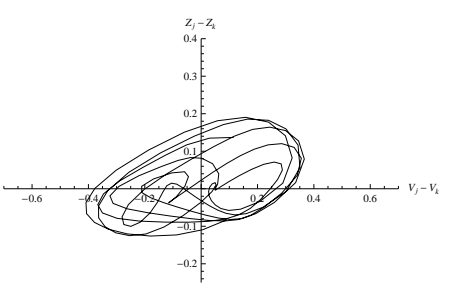

(1) $C=1.2, \tau=10$

Fig. 10. Computational solution curves for the inhibitory node potential differences versus pyramidal node potential differences for different values of $\tau$. Note that the first column of figures contains graphs for $C=0.8$, the middle column contains graphs for $C=1$, and the rightmost column contains graphs for $C=1.2$. The four rows of figures correspond to the four values of $\tau$ investigated $(1,2,5$, and $10 \mathrm{~s})$. The time periods correspond to those used in Figures 3 , 4 , and 5 . 


\section{CONCLUSIONS}

A model describing the change in membrane potential in excitatory and inhibitory neurons in the CA3 region of the hippocampus [25] was modified to more explicitly contain a parameter representing membrane capacitance. The revised model uses ordinary differential equations for both excitatory cells $\left(V_{i}\right)$ and for inhibitory cells $\left(Z_{i}\right)$, and revisions included adapting equations for $Z_{i}$ to be more similar to those for $V_{i}$. The revised model also incorporates delay differential equations to allow for signaling between nodes. A smaller lattice size was used in the current investigation than in the previous model [25], and variation between nodes does appear to increase with lattice size as shown in Figure 2.

In order to ascertain the importance of an explicitly defined capacitance parameter, model output was generated for various values of $C$ and $\tau$. As shown in Figures 3 10, values of $C$ above and below 1 result in different model output for values of $V_{i}$ and $Z_{i}$ and affect the level of synchronization of the lattice the model predicts.

The model output is sensitive to changes in the value of $C$ (for various values of $\tau$ ) which indicates that the accuracy of this parameter does affect model predictions. Hence, these results show how critical the choice of $C$ is because $C=0.8$ may be more realistic a value for $C$ for hippocampal neurons than the more commonly used $C=1$ [36]. Model output is also affected by values of $\tau$, with larger values of $\tau$ resulting in more oscillation.

Assumptions were made in the process of generating simulations, such as choosing similar parameters for inhibitory and excitatory nodes. Parameters such as channel conductances may vary and may affect model output [24]. In this study, all parameters were fixed except for $C$ and $\tau$ in the local, visual sensitivity analysis. Because the sensitivity analysis was local and visual, changes in other fixed parameters may affect output results. However, changes in model predictions were seen for different values of $C$ which shows that for some sets of parameters, the model does depend on the value of $C$, and hence, the results indicate that $C$ is important to accurate model output. The model also appears to be sensitive to the parameter $\tau$ and additional investigation on that parameter may be necessary as well.

Another aspect of the current study that may affect simulation output is the size of the lattice. The lattice used in the current study had fewer nodes than in the Larter model [25]. Because the nodes represent groups of cells rather than individual neurons, the $20 \times 20$ lattice can be viewed as a coarser representation of the same system. A reduced lattice was also used in the current study for simplicity because the introduction of the delay and modeling inhibitory node communication increased computational difficulty.

The model in the current study describes changes in membrane potential in excitatory and inhibitory cells in the hippocampus and is based on previous models [25], [34], [38]. This system of differential equations is intended to help understand epileptic seizures, and innovative treatment strategies may be developed through this understanding. The model is rooted in the specifics of the Hodgkin and Huxley model [34] and later simplifications of that model [38], but information may be learned about the overall system by using these simplifications [14]. Additionally, the number of elderly patients with epilepsy and seizure disorders is likely increasing but access to relevant tissue samples can be problematic [41]. Predictive mathematical models based on physiological mechanisms are potentially useful in identifying changes with age that affect seizures and hence may be useful in identifying treatment strategies for epileptic patients of various ages. The structure of the model may not reveal all aspects of epilepsy at this point, but more can be learned through refining and further investigation of this system through mathematics. 


\section{ACKNOWLEDGMENTS}

Dr. Yokley and Ms. Rouiller were supported in part by the 2011 Summer Undergraduate Research Experience (SURE) program at Elon University. Dr. Luke was supported in part by the National Science Foundation grant HRD-103699.

\section{REFERENCES}

[1] J. Engel, Jr., Introduction to temporal lobe epilepsy, Epilepsy Research, 26 (1996), 141-150.

[2] M. Glass, M. Dragunow, Neurochemical and morphological changes associated with human epilepsy, Brain Research Reviews, 21 (1995), 29-41.

[3] J.P. Karis, Epilepsy, American Journal of Neuroradiology, 29 (2008), 1222-1224.

[4] N.C. de Lanerolle, T-S. Lee, New facets of the neuropathology and molecular profile of human temporal lobe epilepsy, Epilepsy and Behavior, 7 (2005), 190203.

[5] R. Raedt, A. Van Dycke, K. Vonck, P. Boon, Cell therapy in models for temporal lobe epilepsy, Seizure, 16 (2007), 565-578.

[6] L.M. Li, F. Cendes, C. Watson, F. Andermann, D.R. Fish, F. Dubeau, S. Free, A. Oliver, W. Harkness, D.G.T. Thomas, J.S. Duncan, J.W.A.S. Sander, S.D. Shorvon, M.J. Cook, D.L. Arnold, Surgical treatment of patients with single and dual pathology: Relevance of lesion and hippocampal atrophy, Neurology, 48 (1997), 437-444.

[7] L.M. Li, F. Cendes, F. Andermann, C. Watson, D.R. Fish, M.J. Cook, F. Dubeau, J.S. Duncan, S.D. Shorvon, S.F. Berkovic, S. Free, A. Oliver, W. Harkness, D.L. Arnold, Surgical outcome in patients with epilepsy and dual pathology, Brain, 122 (1999), 799-805.

[8] M.P. Richardson, Epilepsy and surgical mapping, British Medical Bulletin, 65 (2003), 179-192.

[9] V. Salanova, O. Markand, R. Worth, R. Smith, H. Wellman, G. Hutchins, H. Park, B. Ghetti, B. Azzarelli, FDG-PET and MRI in temporal lobe epilepsy: relationship to febrile seizures, hippocampal sclerosis and outcome, Acta Neurologica Scandinavica, 97(3) (1998), 146-153.

[10] M.R. Querol Pascual, Temporal Lobe Epilepsy: Clinical Semiology and Neurophysiological Studies, Seminars in Ultrasound, CT, and MRI, 28(6) (2007), 416-423.

[11] S. Wiebe, A. Gafni, W.T. Blume, J.P. Girvin, An Economic Evaluation of Surgery for Temporal Lobe Epilepsy, Journal of Epilepsy, 8 (1995), 227-235.

[12] S. Wiebe, W.T. Blume, J.P. Girvin, M. Eliasziw, A randomized, controlled trial of surgery for temporal-lobe epilepsy, The New England Journal of Medicine, 345(5) (2001), 311-318.

[13] J. Bélair, L. Glass, U. an der Heiden, J. Milton, Dynamical disease: Identification, temporal aspects and treatment strategies of human illness, Chaos, 5(1) (1995), $1-7$.
[14] A.B. Holt, T.I. Netoff, Computational modeling of epilepsy for an experimental neurologist, Experimental Neurology, In press, corrected proof. http://dx.doi.org/10.1016/j.expneurol.2012.05.003

[15] W.W. Lytton, K.M. Hellman, T.P. Sutula, Computer models of hippocampal circuit changes of the kindling model of epilepsy, Artificial Intelligence in Medicine, $\mathbf{1 3}$ (1998), 81-97.

[16] W.W. Lytton, R. Orman, M. Stewart, Computer simulation of epilepsy: Implications for seizure spread and behavioral dysfunction, Epilepsy \& Behavior, 7, 336344.

[17] R.D. Traub, R.K.S. Wong, Cellular Mechanism of Neuronal Synchronization in Epilepsy, Science, 216(4547) (1982), 745-747.

[18] R.D. Traub, R. Miles, R.K.S. Wong, Model of the Origin of Rhythmic Population Oscillations in the Hippocampal Slice, Science, 243 (1989), 1319-1325.

[19] R.D. Traub, R. Miles, J.G.R. Jefferys, Synaptic and intrinsic conductances shape picrotoxin-induced synchronized after-discharges in the guinea-pig hippocampal slice, Journal of Physiology, 461 (1993), 525-547.

[20] A.T. Berg, J. Lin, N. Ebrahimi, F.M. Testa, S.R. Levy, S. Shinnar, Modeling remission and relapse in pediatric epilepsy: application of a Markov process, Epilepsy Research, 60 (2004), 31-40.

[21] K.C. Carlsson, N.O. Hoem, T. Glauser, A.A. Vinks, Development of a Population Pharmacokinetic Model for Carbamazepine Based on Sparse Therapeutic Monitoring Data from Pediatric Patients with Epilepsy, Clinical Therapeutics, 27(5) (2005), 618-626.

[22] A.M. Castelfranco, H.W. Stech, Periodic Solutions in a Model of Recurrent Neural Feedback, SIAM Journal on Applied Mathematics, 47(3) (1987), 573-588.

[23] A. Destexhe, Oscillations, complex spatiotemporal behavior, and information transport in networks of excitatory and inhibitory neurons, Physical Review E, 50(2) (1994), 1594-1606.

[24] D. Golomb, J. Rinzel, Dynamics of globally coupled inhibitory neurons with heterogeneity, Physical Review $E$, 48(6) (1993), 4810-4814.

[25] R. Larter, B. Speelman, R.M. Worth, A coupled ordinary differential equation lattice model for the simulation of epileptic seizures, Chaos, 9(3) (1999), 795-804.

[26] R.E. Plant, A Fitzhugh Differential-Difference Equation Modeling Recurrent Neural Feedback, SIAM Journal on Applied Mathematics, 40(1) (1981), 150-162.

[27] C.L. Stokes, J. Rinzel, Diffusion of Extracellular K+ Can Synchronize Bursting Oscillations in a Model Islet of Langerhans, Biophysical Journal, 65 (1993), $597-$ 607.

[28] H.R. Wilson, J.D. Cowan, Interactions in localized populations of model neurons, Biophysical Journal, 12 (1972), 1-24.

[29] A. Babloyantz, A. Destexhe, Low-dimensional chaos in an instance of epilepsy, Proceedings of the National Academy of Sciences, 83 (1986), 3513-3517. 
K. A. Yokley et al., Computational Sensitivity Analysis on a Mathematical Model ...

[30] S.N. Jespersen, C.D. Kroenke, L. Østergaard, J.J.H. Ackerman, D.A. Yablonskiy, Modeling dendrite density from magnetic resonance diffusion measurements, $\mathrm{Neu}$ rolmage, 34 (2007), 1473-1486.

[31] J.A.S. Kelso, A. Fuchs, Self-organizing dynamics of the human brain: Critical instabilities and Sil'nikov chaos, Chaos, 5(1) (1995), 65-69.

[32] T.I. Netoff, R. Clewley, S. Arno, T. Keck, J.A. White, Epilepsy in Small-World Networks, Journal of Neuroscience, 24(37) (2004), 8075-8083.

[33] A.L. Hodgkin, A.F. Huxley, The components of membrane conductance in the giant axon of loligo, The Journal of Physiology, 116 (1952), 473-496.

[34] A.L. Hodgkin, A.F. Huxley, A quantitative description of membrane current and its application to conduction and excitation in nerve, The Journal of Physiology, 117 (1952), 500-544.

[35] Mondofacto (http://www.mondofacto.com/facts/dictionary ?membrane+capacitance)

[36] L.J. Gentet, G.J. Stuart, J.D. Clements, Direct Measurement of Specific Membrane Capacitance in Neurons,
Biophysical Journal, 79 (2000), 314-320.

[37] J. Golowasch, G. Thomas, A.L. Taylor, A. Patel, A. Pineda, C. Khalil, F. Nadim, Membrane Capacitance Measurements Revisited: Dependence of Capacitance Value on Measurement Method in Nonisopotential Neurons, Journal of Neurophysiology, 102 (2009), 21612175.

[38] C. Morris, H. Lecar, Voltage oscillations in the barnacle giant muscle fiber, Biophysics Journal, 35(1) (1981), 193-213.

[39] K. Kaneko, Overview of coupled map lattices, Chaos, 2(3) (1992), 279-282.

[40] H.T. Banks, D.M. Bortz, A parameter sensitivity methodology in the context of HIV delay equation models, North Carolina State University, Center for Research in Scientific Computation Technical Reports, CRSC-TR02-24 (2002).

[41] I.E. Leppik, K.M. Kelly, L. de Toledo-Morrell, P.R. Patrylo, R.J. DeLorenzo, G.W. Mathern, H.S. White, Basic research in epilepsy and aging, Epilepsy Research, 68S (2006), S21-S37. 